International Journal of Engineering \& Technology, $7(1.3)(2018) 118-120$
International Journal of Engineering \& Technology
Website: www.sciencepubco.com/index.php/IJET
Research paper

\title{
An enhanced voice based chatbot using artificial intelligence
}

\author{
G. Saratha Devi ${ }^{1 *}$, Vinothini. $\mathrm{G}^{2}$, Janani Devi. $\mathrm{C}^{3}$, Jeyachandrakala. $\mathrm{C}^{2}$ \\ ${ }^{1}$ Assistant Professor, Department of Information Technology, Karpagam College of Engineering, Coimbatore \\ ${ }^{2}$ Student, Department of Information Technology, Karpagam College of Engineering, Coimbatore \\ *Corresponding author E-mail: sarathadevi.techno@gmail.com
}

\begin{abstract}
The chatbot application developed using various programming languages with user interface to send input and receive response contains certain boundaries and limitations. This paper proposes a chatbot application for assisting the information regarding the colleges with the use of artificial intelligence. This system has been embedded artificial intelligence to help the user to resolve the questions by providing a human way interactions to identify the sentences and making a decision itself as response to answer a question overcoming the use of programming languages.
\end{abstract}

Keywords: Artificial Intelligence; Chatbot; Voice Recognition.

\section{Introduction}

A computer program that suggest human conversation throw artificial intelligence typically will converse with the real person but applications are being developed in which two applications can communicate with each other. The application will notify the developer if the unimplemented query is asked by user. Those unimplemented query will add to the database. Then the developer will implement those queries cloud based student information system is an artificial algorithm that analysis the students queries and messages. This system has a built artificial intelligence to answer the query of the student. The answers are proper to the user's queries if the user uncover the answer to be invalid. Machine learning is to learn what is happening and react based on history situation etc. A chatbot in javascript with links to smart conversational API's such as all purpose question answering conversation. The practice of cloud involves computing, storage and networking. But with the quality of machine learning infused in the cloud, the capabilities of the cloud will increase enormously. The intellectual cloud happened to be accomplished of learning from the vast amount of data stored in the cloud and to make up guess and consider situations. This will serve as an intelligent platform to perform tasks much resourcefully.

The process would follow an approach which acquires the sign and streams it to a server. The input voice is then routed and a response is created. This process spaces a large processing necessity on the server's processor and memory resources. This restraint is even more apparent when a large number of users are to be simultaneously housed on the system. Voice recognition requires a two part process of capturing and analysis of an input. While the client utilizes the operating system for an input method to acquire a sign, it is for the client to interpret the signal. This process can alleviate processing from the server and allow the server to generate responses faster than when it has more voice processing requests. The core focus is to improve the information output by producing a response that is relevant to the request, factual and personal. This requires aspects to cause informative and user specific responses.

\section{Related work}

H. Al-Zubaide et al. [2011] proposed "new ontology based approach OntBot uses suitable mapping technique to make over ontologies and knowledge into relational database and then use that knowledge to drive its chats. This approach overcomes a number of traditional shortcomings namely the need to learn and use chatbot specific language such as AIML, and the use of nonmatured technology. OntBot has the additional power of easy users connections using their natural language, and the seamless support of different application domains."

Y. Wu et al. [2008] proposed a classification model based chatbot acquisition method which consists of multiple rough set classifiers that are trained. The results are obtained by voting to the output of these classifiers. The combination of ensemble learning is used to improve the result.

J. du Preez et al [] proposed framework required to support a web service. A black box approach is used to control the communication structure, to and from the web service; the web-service allows all types of clients to communicate to the server from any platform. The provided service is accessible through a produced interface which allows for seamless XML processing and the extensibility increases the lifespan of this service.

\section{Proposed system}

The College assistant system is based on the on the idea of predefined responses. The application picks suitable responses from the repository loaded which is based on the context and query raised by the user. The models built using machine translation methods come with the ability to provide new responses from each word.It enables a very long conversation where the chatbot deals with several user queries. 


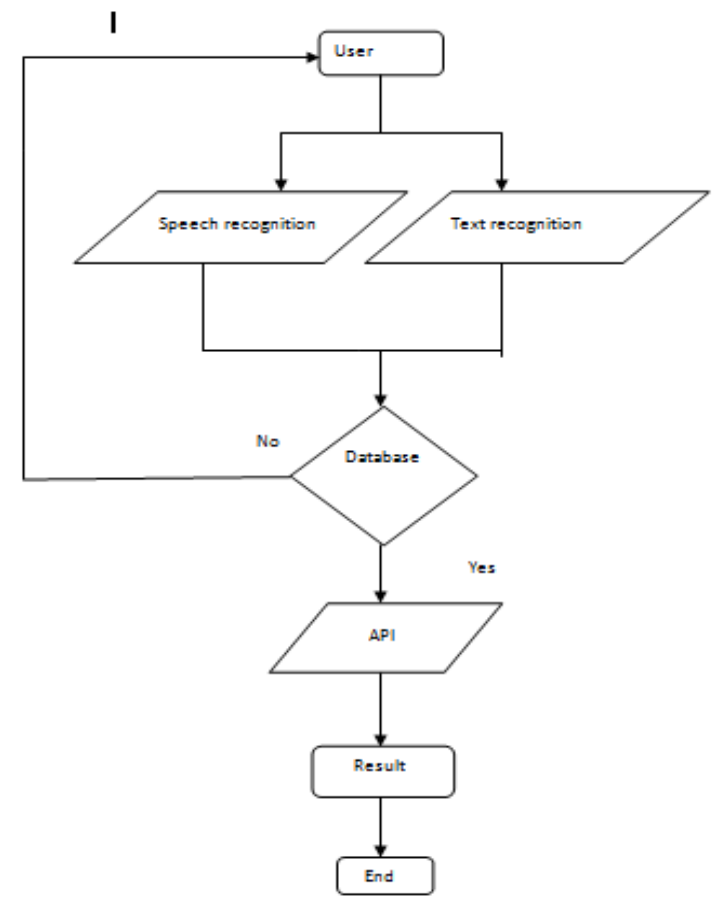

Fig. 1: Data Flow Diagram.

\subsection{Target creation}

The target crated can be included in product or service and convert natural user requests into actionable data. This conversion occurs when a user input matches one of the targets inside your agent. Targets are the predefined or developer-defined components of agents that process a user's request.

The Agents are used to manage a discussion flow in a specific way. This can be done with the help of contexts, target priorities, slot filling, responsibilities, and fulfilment via web clip.

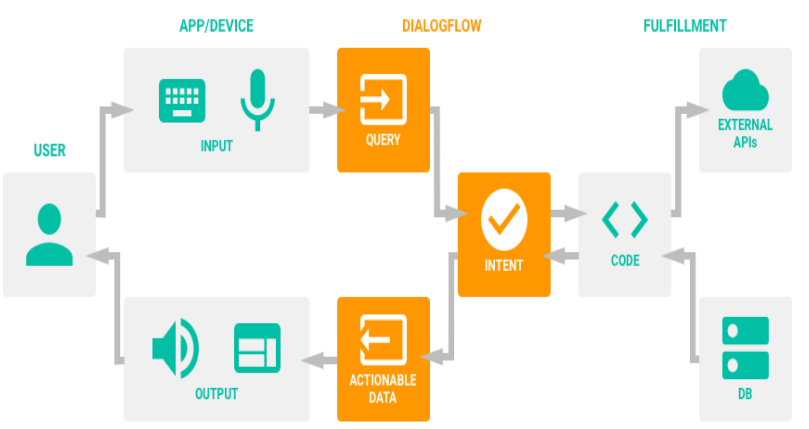

Fig. 2: Target Creation.

\subsection{Request and response}

The application responds and gives appropriate answers depending on user inputs. It also examines and make application to give more than one answer for the same input. This will make chatbot more real, human-like and more reasonable. Each file has two databases. Each database also contains two fields:

1st field: "Request" is where the user inputs are evaluated with data established in this field. See Figures 2 and 3.

2nd field: "Respond" is where the application outputs are taken from data established in this field.
Responding System:

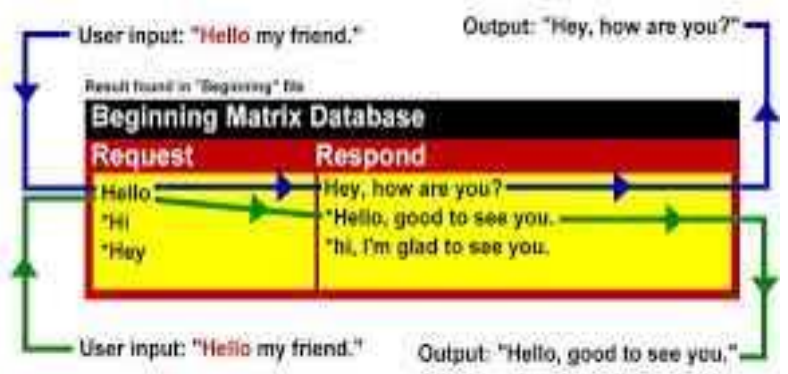

Fig. 3: Request and Response System.

In Figure 2, there are two outputs for the same input. Also, one row can contain more than one request and respond value. This decreases the number of records, as it reduces the repeated sentences in one row.

A variable with an initial value equal one is created. Every time system gives a response, add 1 to the variable value. When the Request value matches with the input given by the user, the output will be equal to the sentence that has the number of the variable. If the variable is enhanced than the last sentence number, make this variable equal to 1 and let the output equal the 1st sentence. Otherwise, add 1 to the variable value.

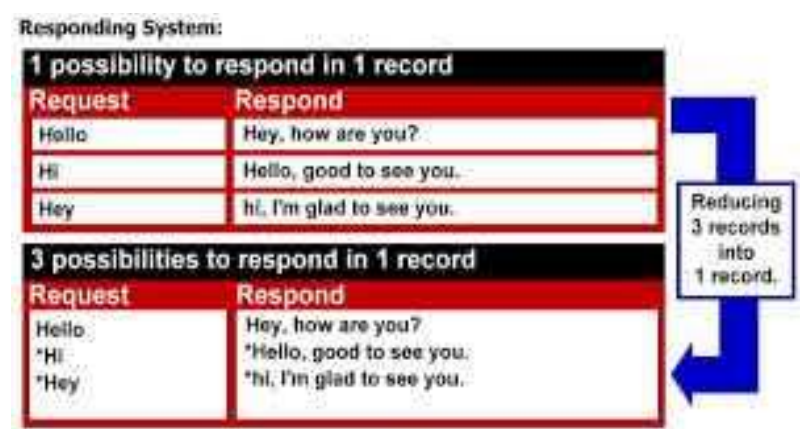

Fig. 4: Merging of Records.

Figure 4 shows how to merge related records into one record. This also leads to merging responses into one record, which makes the application respond in different ways.

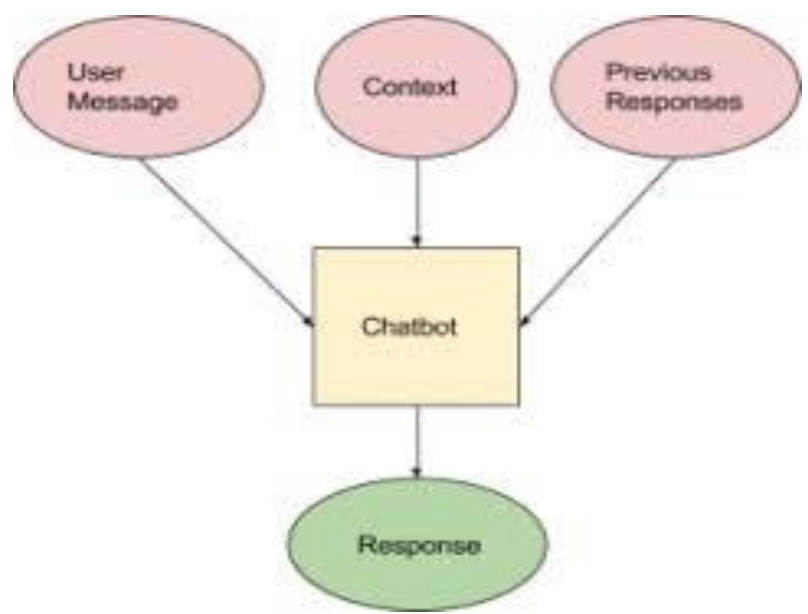

Fig. 5: Request and Response System.

\subsection{Database connectivity}

Fallback intents are triggered if the user input is not matched by any of our regular intents. The questions will be stored in database .Administrator can make modifications and add the responses to these questions in API.ai console. 


\section{Results and discussion}

The college assistant implemented is shown below .The user can interact through the interface shown in the below image. There are five ways of interactions used such as send, speak, clearchat, delete, Admin.

1) Send: In this process, the user can send the queries in the form of text messages to the system. The administrator responds immediately in the form of voice and text message back to the user.

2) Speak: In this process, the user can send the queries in the form of voice messages to the system. The administrator responds immediately in the form of voice message back to the user.

3) Clear chat: The user can clear the chat happened between the administrator and himself.

4) Delete: The administrator have the rights to delete the information or change the information that is no more needed.

5) Admin: The questions asked by the users sometimes if not stored in the database, then it will be send to administrator for immediate response through the website.

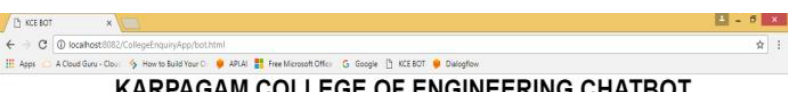

KARPAGAM COLLEGE OF ENGINEERING CHATBOT

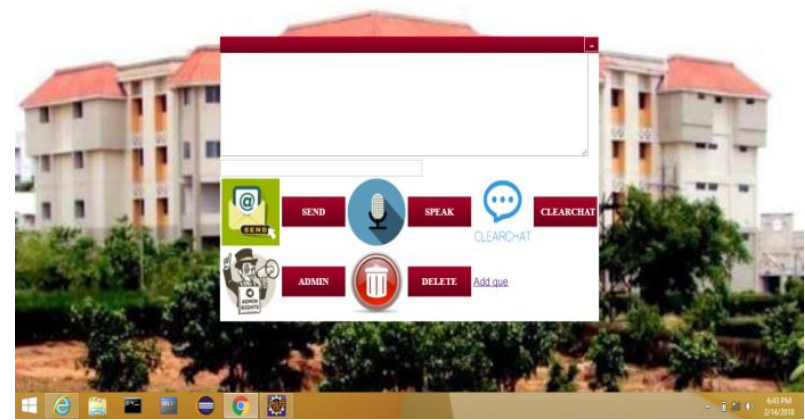

Fig. 6: Sending Request.

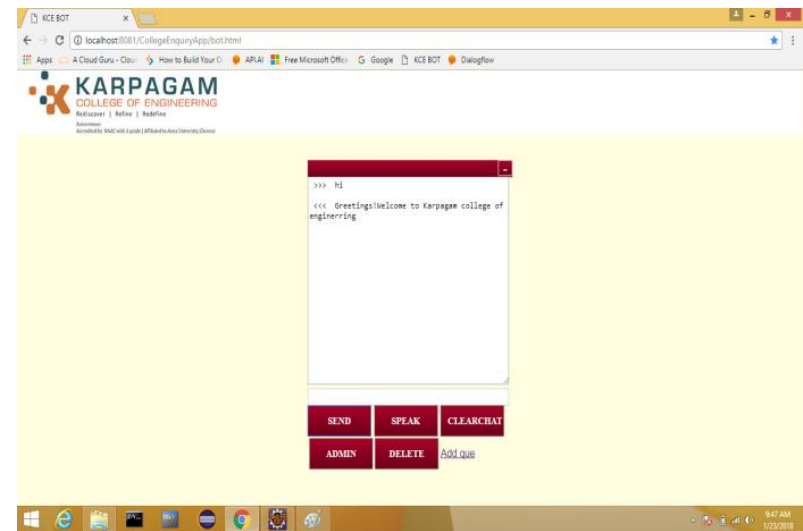

Fig. 7: Receiving Response.

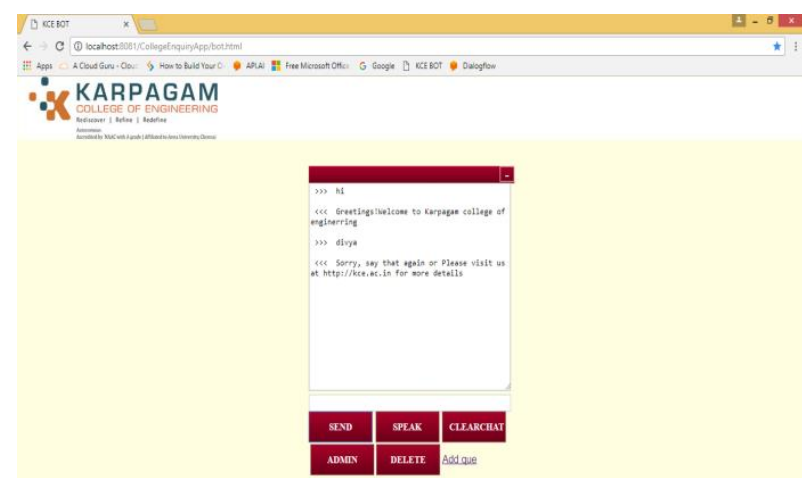

Fig. 8: Immediate Responses.

\section{Conclusion}

Thus the College assistance, a computer program that reproduce human conversation or chat throw artificial intelligence typically a chat bot will communicate with the real person but applications are being developed in which two chatbots can communicate with each other. The chatbot will notify the developer if the unimplemented query is asked by user. Those unimplemented query will add to the database. Then the developer will implement those queries based on intents. This systems has a built artificial intelligence to answer the query of the student. The answers exactly matched to the user's queries if the user find his answer to be invalid he may select the invalid answer option button which will notify the admin. Machine learning is to learn what is happening and react based on history situation etc. A chatbot in javascript with links to smart conversational API's such as all-purpose question answering conversation.

\section{References}

[1] Bayu Setiaji, Ferry Wahyu Wibowo,"Chatbot Using A Knowledge in Database (Human-to-Machine Conversation Modeling)," 2016 7th International Conference on Intelligent Systems, Modelling and Simulation. https://doi.org/10.1109/ISMS.2016.53.

[2] A. Augello, G. Pilato, A. Machi, and S. Gaglio, "An Approach to Enhance Chatbot Semantic Power and Maintainability: Experinces within the FRASI Project," Proc. of 2012 IEEE Sixth International Conference on Semantic Computing, 2012, pp. 186-193, https://doi.org/10.1109/ICSC.2012.26.

[3] H. Al-Zubaide and A. A. Issa, "OntBot: Ontology Based Chatbot," Proc. IEEE of 2011 Fourth International Symposium on Innovation in Information \& Communication Technology (ISIICT), 2011, pp. 7-12, https://doi.org/10.1109/ISIICT.2011.6149594.

[4] J. P. McIntire, L. K. McIntire, and P. R. Havig, "Methods for Chatbot Detection in Distributed Text-Based Communications," Proc. IEEE of 2010 International Symposium on Collaborative Technologies and Systems (CTS), 2010, pp. 463-472, https://doi.org/10.1109/CTS.2010.5478478.

[5] G.Saratha Devi, B.P.Sreeja, "A SURVEY ON INFORMATION CENTRIC NETWORKS FOR COMMUNICATION IN INTER NET OF THINGS" International Journal of Pure and Applied Mathematics, 2018, pp 379-384.

[6] Y. Wu, G. Wang, W. Li, and Z. Li, "Automatic Chatbot Knowledge Acquisition from Online Forum via Rough Set and Ensemble Learning," Proc. IEEE of 2008 IFIP International Conference on Network and Parallel Computing, 2008, pp. 242-246, https://doi.org/10.1109/NPC.2008.24.

[7] A. Augello, M. Scriminaci, S. Gaglio, and G. Pilato, "A Modular Framework for Versatile Conversational Agent Building," Proc. IEEE of 2011 International Conference on Complex, Intelligent and Software Intensive Systems (CISIS), 2011, pp. 577-582, https://doi.org/10.1109/CISIS.2011.95.

[8] G. Pilato, A. Augello, and S. Gaglio, "A Modular Architecture for Adaptive Chatbots," Proc. IEEE of 2011 Fifth IEEE International Conference on Semantic Computing (ICSC), 2011, pp. 177-180, https://doi.org/10.1109/ICSC.2011.68.

[9] G. Neubig, "NLP Programming Tutorial 2 - Bigram Language Models," Presentation Module of Nara Institute of Science and Technology (NAIST).

[10] B.P.Sreeja, L.Jayakumar, G.Saratha Devi, "WIRELESS SENSOR NETWORK APPLICATIONS: A STUDY” International Journal of Pure and Applied Mathematics, 2018, pp 385-389.

[11] M. Dickinson, "Smoothing," Presentation Module of Dept. Of Linguistics, Indiana University, fall 2009.

[12] Y. Bin, P. Cunlin, and L. Dan, "Chinese Text Feature Extraction Method Based on Bigram," Proc. IEEE of 2013 International Communications, Circuits and Systems (ICCCAS), 2013, pp. 342346, https://doi.org/10.1109/ICCCAS.2013.6765352.

[13] J. du Preez, M. Lall and S. Sinha,"AN INTELLIGENT WEBBASED VOICE CHAT BOT" IEEE Conference, 2009, pp. 404 409 978-1-4244-3861-7. https://doi.org/10.1109/EURCON.2009.5167660. 